\title{
LOWER BOUNDS FOR THE WEAK TYPE $(1,1)$ ESTIMATE FOR THE MAXIMAL FUNCTION ASSOCIATED TO CUBES IN HIGH DIMENSIONS
}

\author{
A.S. IAKOVleV AND J.-O. StrömberG
}

\begin{abstract}
In this paper, we will provide the quantitative estimation for the dependence of a lower bound of the Hardy-Littlewood maximal function. This work was inspired by the paper [1] of Stein and Strömberg where general properties of the maximal function were studied. In that work, the increase with the dimension $d$ of the constant $A_{d}$ that appears in the weak type $(1,1)$ inequality for the maximal function was proved however no estimation were given. In a recent paper [2], J.M. Aldaz showed that the lowest constant $A_{d}$ tends to infinity as the dimension $d \rightarrow \infty$. In this paper, we improve the result of J.M. Aldaz providing quantitative estimation of $A_{d} \geq C d^{1 / 4}$, where $C$ is a constant independent of $d$.
\end{abstract}

\section{Introduction}

By a cube $Q(x, r)$ we mean a closed $l_{\infty}$ ball of the radius $r$ with the center at $x$ in $\mathbb{R}^{d}$, in other words $Q(x, r)$ is a closed cube centered at $x$ with sides parallel to coordinate axes, and the side length $2 r$.

Definition. Let $f(x)$ be a function of $L_{1}\left(\mathbb{R}^{d}\right)$ then the maximal function $M_{d} f(x)$ associated with the cube $Q(x, r)$ is defined as follows

$$
M_{d} f(x):=\sup _{r>0} \frac{1}{|Q(x, r)|} \int_{Q(x, r)}|f(y)| d y,
$$

where $|Q(x, r)|$ stands for the Lebesgue measure in $\mathbb{R}^{d}$. In this paper, it will be convenient to use the following representations for this quantity:

$$
|Q(x, r)|=\int_{Q(x, r)} 1 d y=\int_{\mathbb{R}^{d}} \chi_{Q(x, r)}(y) d y .
$$

Here and in what follows $\chi_{Q}$ denotes the characteristic function of the set $Q$.

A fundamental property of the Hardy-Littlewood maximal function $M_{d}$ consists in the fact that it satisfies the weak-type $(1,1)$ inequality, i.e., there exists a constant $A>0$, such that for all $\alpha>0$ and for all $f \in L_{1}$

$$
\alpha\left|\left\{x: M_{d} f(x) \geq \alpha\right\}\right| \leq A\|f\|_{L_{1}} .
$$

Let us denote by $A_{d}$ the best (i.e., lowest) constant $A$ satisfying this inequality in $\mathbb{R}^{d}$.

Received by the editors November 5, 2012. 


\section{Result}

The result of this paper is formulated in the following theorem

Theorem: If $M_{d}$ is a maximal function defined in (1.1) and weak-type inequality (1.3) holds then there exists a constant $c>0$ independent of $d$ such that $A_{d} \geq c d^{1 / 4}$.

\section{Proof of the theorem}

In course of the proof of the theorem we will use a discrete infinite measure $\mu$ which is constructed as Dirac deltas placed at each point of the integer lattice $\mathbb{Z}^{1}$. Then, for $d$-dimensional space $\mu^{d}$ is defined as $\mu^{d}=\mu^{1} \times \mu^{1} \times \cdots \times \mu^{1}$. That will allow us to prove the theorem utilizing the following three steps. At the first stage we will prove the following proposition

Proposition: Let $Q_{0}$ be the unit cube $[0,1]^{d}$ in $\mathbb{R}^{d}$. Then there exists $\alpha_{0}$ such that

$$
\alpha_{0}\left|\left\{x: x \in Q_{0}, M_{d} \mu^{d}(x)>\alpha_{0}\right\}\right|>c_{0} d^{1 / 4},
$$

where $c_{0}$ is a constant independent of $d$.

The proof of this proposition is the main part of the proof of the theorem formulated above. As an immediate consequence of the proposition we will see that if the proportion holds for $Q_{0}$ then $\mu^{d}$ can be restricted to a cube $Q\left(x, r_{0}\right)$ with radius $r_{0}=\rho d^{1 / 4}, r_{0} \in \mathbb{N}$ and the result of the proposition will still hold.

At the second stage, we will be able to restrict the infinite measure $\mu^{d}$ to a significantly large cube to get a finite measure and then proceed applying the following lemma

Lemma 1. For every dimension $d$ there exists a closed cube $K(R) \in \mathbb{R}^{d}$ with sidelength $R \in \mathbb{Z}$ such that if we restrict $\mu^{d}$ onto $K(R)$ and denote by $N$ the number of translated unit cubes $Q_{0}+n$ where $n \in \mathbb{Z}^{d}$ inside $K(R)$ where the proposition holds and the corresponding cube $Q\left(x, r_{0}\right)+n \subset K(R)$ then

$$
\lim _{d \rightarrow \infty} \frac{N}{(R+1)^{d}}=1 .
$$

Note that $(R+1)^{d}$ is equal to the number of Dirac delta functions within $K(R)$.

The finite measure $\left.\mu^{d}\right|_{K(R)}$ form Lemma 1 then can be used in the mollification procedure [3] to obtain a function $f \in L_{1}$ with a desired property. This will be done at the third stage of the proof applying the following lemma

Lemma 2. Let $c$ be a given constant. If there exists a finite sum $\mu=\sum_{i=1}^{n} \delta_{x_{i}}$ of Dirac Deltas in $\mathbb{R}^{d}$ such that the inequality $\alpha\left|\left\{x: M_{d} \mu(x) \geq \alpha\right\}\right|>$ cn is satisfied for some $\alpha>0$, then there exists a function $f \in L^{1}\left(\mathbb{R}^{d}\right)$ and a constant $\beta>0$ for which the inequality $\beta\left|\left\{x: M_{d} f(x) \geq \beta\right\}\right|>c\|f\|_{1}$ holds true.

Technically the most complicated stage is the proof of the proposition. This will be the subject of the next section. We will give the proofs of the Lemmas 1 and 2 in the appendix. 


\section{Proof of the proposition}

4.1. Notations. Let us consider $u \in(0,1 / 2)$ and an interval $I \subset \mathbb{R}$. Let us call $y \in I$ to be centered at level $u$ (more briefly centered) if it belongs to the closed subinterval with the same center as $I$ and with the length $(1-2 u)|I|$ and to be off center (at level $u$ ) otherwise. In particular for $I=[0,1]$, the centered values are those belonging to the interval $[u, 1-u]$. The role of $u$ in the proof is to serve as a parameter to control a placement of the center of our cubes.

Definition. Let $Q_{0}$ be the unit cube $[0,1]^{d}$ in $\mathbb{R}^{d}$ and let $E_{u, k}^{d}$ be a set consisting of $x \in Q_{0}$ such that $k$ coordinates of the vector $x=\left\{x_{1}, \ldots, x_{d}\right\}$ are off-centered at level $u$ whereas remaining $d-k$ coordinates are centered.

It is clear that for each particular choice of $k$ off-centered coordinates the corresponding subset of $E_{u, k}^{d}$ has the measure $(2 u)^{k}(1-2 u)^{d-k}$. For the entire $E_{u, k}^{d}$ with combinatorial arguments one gets

$$
\left|E_{u, k}^{d}\right|=(2 u)^{k}(1-2 u)^{d-k}\left(\begin{array}{l}
d \\
k
\end{array}\right),
$$

where $\left(\begin{array}{l}d \\ k\end{array}\right)$ stands for the binomial coefficient.

Let $x$ be a point in the set $E_{u, k}^{d}$ and let $s$ be a positive integer $s>0$, then the cube $Q(x, s-u)$ contains $(2 s-1)^{k}(2 s)^{d-k}$ integer grid points. For this cube, the quantity

$$
M(d, u, k, s)=\frac{1}{|Q(x, s-u)|} \int_{Q(x, s-u)} d \mu^{d}
$$

can easily be computed explicitly

$$
M(d, u, k, s)=\frac{(2 s-1)^{k}(2 s)^{d-k}}{2^{d}(s-u)^{d}} .
$$

The inequality

$$
M_{d} \mu^{d}(x) \geq M(d, u, k, s)
$$

is an obvious consequence of the definition of the maximal function applied to the measure $\mu^{d}$. If now we restrict $x$ onto the set $E_{u, k}^{d}$ then the value of $M(d, u, k, s)$ can be taken as the constant $\alpha_{0}$ in the proposition and the left-hand side of (3.1) in this case takes the explicit form

$$
M(d, u, k, s)\left|\left\{x \in E_{u, k}^{d}: M_{d} \mu^{d}(x) \geq M(d, u, k, s)\right\}\right|=M(d, u, k, s)\left|E_{u, k}^{d}\right| .
$$

In order to expand (4.2) onto entire cube $Q_{0}$ we consider the following construction. Let us take sets $E_{u(k), k}^{d}$ with $k \in \Delta=\left[k_{\min }, k_{\max }\right]$ and $u(k)$ such that $u(j)<u(k)$ if $j<k$. It is apparent that the sum of these sets

$$
Q_{\Delta}=\bigcup_{k \in \Delta} E_{u(k), k}^{d}
$$

will obey the exhausting condition

$$
\lim _{k_{\min } \rightarrow 0} \lim _{k_{\max } \rightarrow d} Q_{\Delta}=Q_{0} .
$$


Then, in order to expand (4.2) onto the set $Q_{\Delta}$ one needs to take the minimal value of all $M(d, u(k), k, s)$ terms, i.e.,

$$
M_{0}=\min _{k \in \Delta} M(d, u(k), k, s) .
$$

As the result the extension of the relation (4.2) onto $Q_{\Delta}$ reads as

$$
M_{0}\left|\left\{x \in Q_{\Delta}: M_{d} \mu^{d}(x) \geq M_{0}\right\}\right|=M_{0}\left|Q_{\Delta}\right|,
$$

where $M_{0}$ now serves as the constant $\alpha_{0}$ of the proposition. This observation is the basis for all estimations that follows. In order to prevent overloading of formulae below we will often suppress in notations the dependence of $u$ on $k$ implying it implicitly.

As the next step we will estimate the value of $\left|Q_{\Delta}\right|$. Let us make use of the representation (1.2) and use the Schwarz inequality in the form

$$
\left[\int \chi_{Q_{\Delta}} \sum_{k \in \Delta} \chi_{E_{u, k}^{d}} d x\right]^{2} \leq \int \chi_{Q_{\Delta}}^{2} d x \int \sum_{k \in \Delta} \chi_{E_{u, k}^{d}} \sum_{j \in \Delta} \chi_{E_{u, j}^{d}} d x .
$$

Taking into account the property of the characteristic function $\chi_{Q_{\Delta}}^{2}=\chi_{Q_{\Delta}}$, the obvious identity

$$
\chi_{Q_{\Delta}} \sum_{k \in \Delta} \chi_{E_{u, k}^{d}}=\sum_{k \in \Delta} \chi_{E_{u, k}^{d}}
$$

and properly rearranging the terms in the double sum of the integrand in the second integral of the right-hand side of (4.4) we arrive at the inequality

$$
\left|Q_{\Delta}\right|=\left(\int \chi_{Q_{\Delta}} d x\right) \geq \frac{\left(\int \sum_{k} \chi_{E_{u, k}^{d}} d x\right)^{2}}{\int \sum_{k} \chi_{E_{u, k}^{d}} d x+2 \int \sum_{k>j} \chi_{E_{u, k}^{d} \cap E_{u, j}^{d}} d x} .
$$

Here it is assumed that the $k$ and $j$ sums are taken over the interval $\Delta$.

Introducing (4.5) into (4.3) we obtain the inequality

$$
M_{0}\left|Q_{\Delta}\right| \geq \frac{M_{0}^{2}\left(\int \sum_{k} \chi_{E_{u, k}^{d}} d x\right)^{2}}{M_{0} \int \sum_{k} \chi_{E_{u, k}^{d}} d x+2 M_{0} \int \sum_{k>j} \chi_{E_{u, k}^{d} \cap E_{u, j}^{d}} d x} .
$$

In we now take into account the monotonic character of $M(d, u, k, s)$ as the function of $u$ and $k$ then this inequality can be strengthened to take the form

$$
M_{0}\left|Q_{\Delta}\right| \geq\left(M_{0} / M_{1}\right)^{2} \frac{\left(\int \sum_{k} M_{k} \chi_{E_{u, k}^{d}} d x\right)^{2}}{\int \sum_{k} M_{k} \chi_{E_{u, k}^{d}} d x+2 \int \sum_{k>j} M_{k} \chi_{E_{u, k}^{d} \cap E_{u, j}^{d}} d x} .
$$

Here for shortening of notations, we have introduced $M_{1}=\max _{k \in \Delta} M(d, u, k, s)$ and $M_{k}=M(d, u, k, s)$.

In what follows, we will deal with this representation. The right-hand side of (4.7) will be estimated by making use of the appropriate estimations for every term of this fraction. For that purpose, we will look for $s_{0}$ such that $M\left(d, u, k, s_{0}\right)=$ $\sup _{s \in \mathbb{R}_{+}} M(d, u, k, s)$. In principle, $s_{0}$ should be integer, i.e., $s_{0} \in \mathbb{N}$, however technically the case of real value of $s_{0}$ such that $s_{0} \in \mathbb{R}_{+}$can be easier analyzed. From now on we will consider parameter $s$ to be real valued. The latter will allow us using the differential calculus for computing extrema. 
In order to be able to use the same estimations for $s_{0}$ real and integer we will need $\sup _{s \in \mathbb{R}_{+}} M(d, u, k, s)$ and $\sup _{s \in \mathbb{Z}_{+}} M(d, u, k, s)$ to have the same order of $d$. That will be a restriction on some of our parameters. Formally this restriction can be written as

$$
\frac{\sup _{s \in \mathbb{R}_{+}} M(d, u, k, s)}{\sup _{s \in \mathbb{Z}_{+}} M(d, u, k, s)}<e .
$$

In general, it is enough to have any constant independent of $d$ in the right-hand side of this inequality. This special choice of the bound is made for computational convenience and will be used later.

4.2. Estimations for $s \in \mathbb{R}$. We start by finding $s_{0}$ that provides the maximal value for $M_{k}$. The problem reduces to find the solution of the equation

$$
\frac{\partial}{\partial s} \log M(d, u, k, s)=\frac{(d-k) u+(k-2 u d) s}{(2 s-1) s(s-u)}=0,
$$

which yields

$$
s_{0}=(d-k) u /(2 u d-k) .
$$

This expression introduces the following restriction for $k$, i.e., since the value of $s_{0}$ is sufficiently positive, $k$ should not exceed $2 u d$, i.e., $k<2 u d$. Calculating $M_{k}$ at $s=s_{0}$ leads us to the following result

$$
M\left(d, u, k, s_{0}\right)=\frac{k^{k}(d-k)^{d-k}}{d^{d}(2 u)^{k}(1-2 u)^{d-k}} .
$$

We can now fix the parameter $s$ in all above and following formulae as $s=s_{0}$. Let us estimate the integral

$$
\int M_{k} \chi_{E_{u, k}^{d}} d x
$$

from (4.7) when $d \gg 1$ and $k \gg 1$ recalling that

$$
\int M_{k} \chi_{E_{u, k}^{d}} d x=M\left(d, u, k, s_{0}\right)\left|E_{u, k}^{d}\right|
$$

Whereas the first factor in the right-hand side has the relatively simple form (4.10), the second one contains the binomial coefficient (4.1).

This coefficient can be evaluated for large values of $d$ and $k$ by using the Stirling formula [4]

$$
n !=\sqrt{2 \pi n}\left(\frac{n}{e}\right)^{n}(1+O(1 / n)) .
$$

In our construction we will restrict $k$ to be in the interval $[\zeta d, \xi d]$, where $0<\zeta<\xi<1$ which implies that the lower bound for $k$, namely $k_{\text {min }}$, in our computations will also be considered large.

Therefore, the binomial coefficient $\left(\begin{array}{l}d \\ k\end{array}\right)$ in (4.1) can be approximated as

$$
\left(\begin{array}{l}
d \\
k
\end{array}\right) \approx \frac{\sqrt{d}}{\sqrt{2 \pi(d-k) k}} \frac{d^{d}}{k^{k}(d-k)^{d-k}},
$$


which yields

$$
M\left(d, u, k, s_{0}\right)\left|E_{u, k}^{d}\right| \approx \frac{\sqrt{d}}{\sqrt{2 \pi(d-k) k}} .
$$

Consequently, for the sum of such terms we get

$$
\int \sum_{k \in \Delta} M_{k} \chi_{E_{u, k}^{d}} d x=\sum_{k \in \Delta} M\left(d, u, k, s_{0}\right)\left|E_{u, k}^{d}\right| \approx \sum_{k \in \Delta} \frac{\sqrt{d}}{\sqrt{2 \pi(d-k) k}} .
$$

For large $d$ the sum in the right-hand side of (4.12) can be transformed to an expression containing the Riemann integral sum, i.e.,

$$
\sum_{k \in \Delta} \frac{\sqrt{d}}{\sqrt{2 \pi(d-k) k}}=d^{1 / 2} \sum_{k \in \Delta} \frac{1 / d}{\sqrt{2 \pi(1-k / d) k / d}}
$$

for the integral

$$
I(\zeta, \xi)=\int_{\zeta}^{\xi} \frac{d \tau}{\sqrt{2 \pi(1-\tau) \tau}},
$$

where $\zeta=k_{\min } / d$ and $\xi=k_{\max } / d$. This implies that

$$
\int \sum_{k \in \Delta} M_{k} \chi_{E_{u, k}^{d}} d x \approx d^{1 / 2} I(\zeta, \xi) .
$$

The integral $I(a, b)$ in the right-hand side of (4.14) converges for all $a$ and $b$ such that $(a, b) \in(0,1)$. Now it is obvious that, since the integrand in (4.13) is positive, then extending or narrowing of the integration interval $(a, b)$ relatively to $(\zeta, \xi)$ will lead us to the upper or lower bounds. So that we can state that there exist constants $B_{1}<B_{2}$, which are independent of $d$ such that

$$
B_{1} d^{1 / 2} \leq \int \sum_{k \in \Delta} M_{k} \chi_{E_{u, k}^{d}} d x \leq B_{2} d^{1 / 2} .
$$

Let us notice that in any case $B_{2}$ can be taken as $I(0,1)$. These inequalities allow us to estimate the numerator term and the first term in denominator of (4.7).

What is left is the estimation of the second term in the denominator of the fraction in (4.7).

Recalling that $u=u(k)$ and $u(k)>u(j)$ if $k>j$ and setting for shortening of notations $u(k)=u$ and $u(j)=v$, the measure of the intersection of two sets $E_{u, k}^{d}$ and $E_{v, j}^{d}$ can easily be evaluated as

$$
\left|E_{u, k}^{d} \cap E_{v, j}^{d}\right|=(2 v)^{j}(2 u-2 v)^{k-j}(1-2 u)^{d-k}\left(\begin{array}{l}
d \\
k
\end{array}\right)\left(\begin{array}{l}
k \\
j
\end{array}\right) .
$$

After regrouping the factors we arrive at the expression

$$
\left|E_{u, k}^{d} \cap E_{v, j}^{d}\right|=(2 u)^{k}(1-2 u)^{d-k}\left(\begin{array}{l}
d \\
k
\end{array}\right)(2 w)^{j}(1-2 w)^{k-j}\left(\begin{array}{l}
k \\
j
\end{array}\right),
$$

where $w=v / 2 u$. Using (4.11) for $\{d, k, j\} \gg 1$ we get

$$
\left|E_{u, k}^{d} \cap E_{v, j}^{d}\right| \approx\left|E_{u, k}^{d}\right| \frac{\sqrt{k}}{\sqrt{2 \pi(k-j) j}} M^{-1}\left(k, w, j, s_{0}\right)
$$


and consequently

$$
M\left(d, u, k, s_{0}\right)\left|E_{u, k}^{d} \cap E_{v, j}^{d}\right| \approx \frac{\sqrt{d}}{\sqrt{2 \pi(d-k) k}} \frac{\sqrt{k}}{\sqrt{2 \pi(k-j) j}} M^{-1}\left(k, w, j, s_{0}\right) .
$$

Introducing representations derived in (4.15) into the right-hand side of (4.7) we obtain

$$
M_{0}\left|Q_{\Delta}\right| \geq \frac{\left(\sum_{k} \frac{\sqrt{d}}{\sqrt{2 \pi(d-k) k}}\right)^{2}}{\sum_{k} \frac{\sqrt{d}}{\sqrt{2 \pi(d-k) k}}+2 \sum_{k>j} \frac{\sqrt{d}}{\sqrt{2 \pi(d-k) k}} \frac{\sqrt{k}}{\sqrt{2 \pi(k-j) j}} M^{-1}\left(k, w, j, s_{0}\right)} .
$$

Now we rewrite the double sum in the form

$$
\begin{aligned}
& \sum_{k>j} \frac{\sqrt{d}}{\sqrt{2 \pi(d-k) k}} \frac{\sqrt{k} M^{-1}\left(k, w, j, s_{0}\right)}{\sqrt{2 \pi(k-j) j}} \\
& \quad=\sum_{k \in \Delta} \frac{\sqrt{d}}{\sqrt{2 \pi(d-k)}} \sum_{j=k_{\text {min }}}^{k-1} \frac{M^{-1}\left(k, w, j, s_{0}\right)}{\sqrt{2 \pi(k-j) j}} .
\end{aligned}
$$

As in the case of the sum (4.12) the double sum (4.17) can be transformed into the integral sum of some double integral. This transformation will be the subject of the rest of this subsection and finally will provide us with the desired estimation for the second term in the denominator of (4.16). First we estimate $M^{-1}\left(k, w, j, s_{0}\right)$.

For computational convenience, we introduce a new function $L(k, w, j)$ defined as follows:

$$
L(k, w, j)=\log M\left(k, w, j, s_{0}\right) .
$$

To get an estimation for $L(k, w, j)$ from below we will construct the Taylor expansion with respect to $j$ around minimal value of this function. From here on we will treat both $j$ and $k$ as real parameters which will allow us to calculate derivatives and determine dependance for other parameters. This procedure does not affect the accuracy since at every moment the restriction from $\mathbb{R}$ to $\mathbb{Z}$ and be performed.

Then the value of $j_{0}$ that provides the minimum to $L(k, w, j)$ can be found from the following equation:

$$
\frac{\partial}{\partial j} L(k, w, j)=\log \left(\frac{j(1-2 w)}{2 w(k-j)}\right)=0
$$

and has the form $j_{0}=2 w k$. By direct computation one gets $L\left(k, w, j_{0}\right)=0$. f derivative of $L(k, w, j)$ with respect to $j$ has the following form:

$$
\frac{\partial^{2}}{\partial j^{2}} L(k, w, j)=\frac{k}{j(k-j)} \geq 0,
$$

which shows that the Taylor expansion up to the first nonzero term of second order can be written as follows:

$$
L(k, w, j)=\frac{1}{2} \frac{\left(j_{0}-j\right)^{2}}{2 w k(1-2 w)}+O\left(\left(j_{0}-j\right)^{3}\right) .
$$

The value of $j_{0}-j$ in the numerator can be represented as

$$
j_{0}-j=2 w k-j=\frac{u(j)}{u(k)} k-j=u(j)\left(\frac{k}{u(k)}-\frac{j}{u(j)}\right) .
$$


By the Mean value theorem we obtain

$$
\frac{k}{u(k)}-\frac{j}{u(j)}=\frac{\partial}{\partial k}\left(\frac{k}{u(k)}\right)_{k=\tilde{k}}(k-j)
$$

where $\tilde{k} \in(j, k)$. The value $\frac{\partial}{\partial k}\left(\frac{k}{u(k)}\right)$ can be evaluated using the following considerations. By Implicit function theorem we define $u(k)$ as the solution of the equation

$$
L(d, u, k)=L_{0},
$$

where $L_{0}=\log \left(M_{0}\right)$ is a constant. Then differentiating (4.19) and using

$$
\frac{d}{d k} L(d, u(k), k)=\frac{\partial}{\partial k} L(d, u(k), k)+\frac{\partial}{\partial u} L(d, u(k), k) \frac{\partial u(k)}{\partial k}
$$

we arrive at the representation

$$
\frac{\partial u(k)}{\partial k}=-\frac{\frac{\partial}{\partial k} L(d, u(k), k)}{\frac{\partial}{\partial u} L(d, u(k), k)},
$$

or more explicitly

$$
\frac{\partial u(k)}{\partial k}=-\frac{\log \left(\frac{k(1-2 u)}{2 u(d-k)}\right)}{\frac{2 d u-k}{(1-2 u) u}} .
$$

Consequently for $\frac{\partial}{\partial k}\left(\frac{k}{u(k)}\right)$ we get

$$
\frac{\partial}{\partial k}\left(\frac{k}{u(k)}\right)=\frac{1}{u}+\frac{k(1-2 u)}{u(2 u d-k)} \log \left(\frac{k(1-2 u)}{2 u(d-k)}\right) .
$$

To simplify (4.20) will expand the logarithm with help of Taylor series in the vicinity of $k_{0}=2 u d$. Due to the singularity in the prelogarithmic term at $k=k_{0}$ the expansion should be taken up to the quadratic term

$$
\log \left(\frac{k(1-2 u)}{2 u(d-k)}\right)=\frac{k-2 u d}{2 u d(1-2 u)}+\frac{(4 u-1)(k-2 u d)^{2}}{2(2 u d)^{2}(1-2 u)^{2}}+O\left((k-2 u d)^{3}\right) .
$$

Substitution into (4.20) leads us to

$$
\frac{\partial}{\partial k}\left(\frac{k}{u(k)}\right)=\frac{2 u d-k}{4 u^{2} d(1-2 u)}+O\left((k-2 u d)^{2}\right) .
$$

The denominator of the right-hand side of (4.18) can be estimated using the following consideration. The value $w$ was set as $w=u(j) / 2 u(k)$. We can express $u(k)$ with the help of $k_{0}$ as follows:

$$
u(k)=\frac{k_{0}}{2 d}=\frac{k_{0}-k+k}{2 d}=\frac{k}{2 d}+\frac{k_{0}-k}{2 d} .
$$

From our previous considerations $k \in[\zeta d, \xi d]$, so we can conclude that

$$
\frac{k}{2 d} \sim \text { const. }
$$

The restriction (4.8) in the form

$$
\log \left(\frac{M\left(d, u, k, s_{0}\right)}{M\left(d, u, k, s_{*}\right)}\right)<1 .
$$


can be used to estimate $k_{0}-k$ as follows. Since $s_{*} \in \mathbb{Z}$ is the point such that $M\left(d, u, k, s_{*}\right)=\sup _{s \in \mathbb{Z}_{+}} M(d, u, k, s)$, it is obvious that $\left|s_{0}-s_{*}\right|<1$. Now if we will show that for any $\tilde{s} \in\left(\min \left(s_{0}, s_{*}\right), \max \left(s_{0}, s_{*}\right)\right)$ the inequality holds

$$
\left.\frac{\partial}{\partial s} \log M(d, u, k, s)\right|_{s=\tilde{s}}\left(s_{0}-s_{*}\right)<1,
$$

then (4.23) will hold true due to the Mean value theorem. The explicit form of (4.24) reads

$$
\frac{\left(k_{0}-k\right)\left(s_{0}-\tilde{s}\right)\left(s_{0}-s_{*}\right)}{(2 \tilde{s}-1) \tilde{s}(\tilde{s}-u)}<1 .
$$

One can strengthen the last inequality removing the positive factor in the numerator $\left(s_{0}-\tilde{s}\right)\left(s_{0}-s_{*}\right)<1$ which yields

$$
\frac{k_{0}-k}{(2 \tilde{s}-1) \tilde{s}(\tilde{s}-u)}<1 \text {. }
$$

Since $\left|\tilde{s}-s_{0}\right|<1$ and $s_{0} \gg 1$ we can replace the denominator by $2 s_{0}^{3}$. Inserting the explicit value (4.9) of $s_{0}$ into inequality (4.25) we get it in the following form

$$
\frac{\left(k_{0}-k\right)^{4}}{2 u^{3}(d-k)^{3}}<1
$$

Thus we have arrived at the estimation

$$
k_{0}-k<(d-k)^{3 / 4}\left(2 u^{3}\right)^{1 / 4} .
$$

It was shown before that $d-k \sim d^{1}$ and $u \sim d^{0}$ then we may conclude that

$$
k_{0}-k<\hat{k}_{1} d^{3 / 4}
$$

with $\hat{k}_{1}$ being a some constant. This inequality being introduced to (4.9) will generate the fact that $s_{0} \sim d^{1 / 4}$ which is consistent with our assumption $s_{0} \gg 1$.

Now we can rewrite $(4.22)$ as

$$
u(k)=\frac{k}{2 d}+O\left(d^{-1 / 4}\right) .
$$

The latter allows us to use the following asymptotic relation

$$
w \simeq \frac{j}{2 k}
$$

as $d \rightarrow \infty$. Thus, we can write asymptotic equality for the denominator of (4.18)

$$
2 j_{0}\left(k-j_{0}\right)=4 w k(k-2 w k) \simeq 2 \frac{j}{k} k\left(k-\frac{j}{k} k\right)=2 j(k-j)
$$

Taking into account (4.21) and (4.27) the formula (4.18) for large $d$ can be rewritten in the form

$$
L(k, w, j) \simeq \frac{k}{2 j(k-j)}\left(\frac{j}{2 d}\right)^{2}\left[\frac{\left(k_{0}-k\right)^{2}}{(2 u d)^{2}(2 u)^{2}(1-2 u)^{2}}\right]_{k=\tilde{k}}(k-j)^{2} .
$$

In order to get the final estimation from below for $L(k, w, j)$ we proceed as follows. The inequalities

$$
2 u d<d, \quad 2 u(1-2 u)<1 / 4
$$


can be used for the denominator, and the numerator can be estimated with the help of inequality

$$
j>k_{\min } \text {. }
$$

For the estimation of $L$, we are dealing with the smaller value of $k_{0}-k$ chosen the better. However, the estimation should be valid for all range of $k_{0}-k$ including the worst case of $k_{0}-k \sim d^{3 / 4}$, i.e., when it is close to its upper bound (4.26). Hence, we will use the inequality $k_{0}-k>\hat{k}_{0} d^{3 / 4}$, where $\hat{k}_{0}<\hat{k}_{1}$ is a constant. This shows that

$$
L(k, w, j)>2 \frac{k k_{\min } \hat{k}_{0}}{d^{5 / 2}}(k-j) .
$$

With this inequality we are ready to perform the final evaluation of the double sum (4.16). At the first stage for the sum with respect to $j$ in (4.17) we get

$$
\sum_{j=k_{\min }}^{k} \frac{M^{-1}\left(k, w, j, s_{0}\right)}{\sqrt{2 \pi(k-j) j}}<\sum_{j=k_{\min }}^{k-1} \frac{\exp \left(-2 \frac{k k_{\min } \hat{k}_{0}}{d^{5 / 2}}(k-j)\right)}{\sqrt{2 \pi(k-j) k_{\min }}} .
$$

Replacing $j$ with $k_{\min }$ in the denominator in the right-hand side makes this inequality even stronger. Changing the summation index in the right-hand side we obtain

$$
\sum_{j=k_{\min }}^{k-1} \frac{M^{-1}\left(k, w, j, s_{0}\right)}{\sqrt{2 \pi(k-j) j}}<\sqrt{\frac{k}{k_{\min }}} \sum_{r=1}^{k-k_{\min }} \frac{1}{\sqrt{2 \pi}} \sqrt{\frac{k}{r}} \exp \left(-2 \frac{k^{2} k_{\min } \hat{k}_{0}}{d^{5 / 2}} \frac{r}{k}\right) \frac{1}{k}
$$

and we can see that the sum in the right-hand side of (4.28) can now be considered when $k \gg 1$ as the Riemann integral sum for the integral

$$
J\left(1-k_{\min } / k, 0\right)=\int_{0}^{1-k_{\min } / k} \frac{1}{\sqrt{2 \pi \tau}} \exp \left(-2 \frac{k^{2} k_{\min } \hat{k}_{0}}{d^{5 / 2}} \tau\right) d \tau .
$$

That implies that

$$
\sum_{j=k_{\min }}^{k-1} \frac{M^{-1}\left(k, w, j, s_{0}\right)}{\sqrt{2 \pi(k-j) j}}<\frac{d^{5 / 4}}{2 k_{\min } \sqrt{k \hat{k}_{0}}} \Phi\left(\sqrt{\frac{\left(1-k_{\min } / k\right) k^{2} k_{\min } \hat{k}_{0}}{d^{5 / 2}}}\right),
$$

where $\Phi(x)$ stands for error function [4] $\Phi(z)=2 / \sqrt{\pi} \int_{0}^{z} \exp \left\{-x^{2}\right\} d x$.

It is possible to show that the argument of the error function has the order of $d^{1 / 4}$. Therefore, one can extend this argument of the error function to $+\infty$ and taking into account that $\Phi(+\infty)=1$, we end up with

$$
\sum_{k \in \Delta} \frac{\sqrt{d}}{\sqrt{2 \pi(d-k)}} \sum_{j=k_{\min }}^{k-1} \frac{M^{-1}\left(k, w, j, s_{0}\right)}{\sqrt{2 \pi(k-j) j}}<\frac{d^{5 / 4}}{2 k_{\min } \hat{k}_{0}^{1 / 2}} \sum_{k \in \Delta} \frac{\sqrt{d}}{\sqrt{2 \pi(d-k) k}} .
$$

The sum on the right-hand side was already estimated in (4.14) as

$$
\sum_{k \in \Delta} \frac{\sqrt{d}}{\sqrt{2 \pi(d-k) k}}<d^{1 / 2} I(0,1) \text {. }
$$

This results

$$
\sum_{k \in \Delta} \frac{\sqrt{d}}{\sqrt{2 \pi(d-k)}} \sum_{j=k_{\min }}^{k-1} \frac{M^{-1}\left(k, w, j, s_{0}\right)}{\sqrt{2 \pi(k-j) j}}<\frac{d^{3 / 4}}{2 \zeta \hat{k}_{0}^{1 / 2}} I(0,1) .
$$


Here, we have used the fact that $k_{\min }=\zeta d$. So, the upper bound for the second term in the denominator of (4.6) can be written in the form

$$
M_{0} \int \sum_{k>j} \chi_{E_{u, k}^{d} \cap E_{u, j}^{d}} d x<B_{3} d^{3 / 4},
$$

where $B_{3}$ is some constant. Finally, we obtain the inequality for $M_{0}\left|Q_{\Delta}\right|$

$$
M_{0}\left|Q_{\Delta}\right|>\frac{B_{1}^{2} d}{B_{2} d^{1 / 2}+2 B_{3} d^{3 / 4}} \sim c_{0} d^{1 / 4}
$$

which finalizes the proof of the proposition.

\section{Final proof}

Collecting results from the previous sections we have proven by direct construction that the constant $M_{0}$ can be taken as $\alpha_{0}$ of the proposition. We have shown that inequality $M_{d} \mu^{d}>M_{0}$ is fulfilled on the set $Q_{\Delta} \subset Q_{0}$. Moreover, we have proven that there exists a constant $c_{0}$, which is independent of $d$, such that

$$
M_{0}\left|Q_{\Delta}\right|>c_{0} d^{1 / 4} \text {. }
$$

Thus, the proposition has been proven.

Now we can use Lemma 1 in order to extend the proposition onto the class of finite measures, i.e., the restriction of the measure $\mu^{d}$ onto a large cube $K(R)$ with sidelength $R \geq \theta d^{5 / 4+\varepsilon}$ leads us to the inequality

$$
\alpha_{0}\left|\left\{x: M_{d}\left(\left.\mu^{d}\right|_{K(R)}\right)(x) \geq \alpha_{0}\right\}\right|>c_{0} d^{1 / 4}(R+1)^{d} .
$$

The final result follows from the Lemma 2, i.e., there exists a constant $\beta>0$ and $f \in L_{1}$ such that

$$
\beta\left|\left\{x: M_{d} f(x) \geq \beta\right\}\right|>c_{0} d^{1 / 4}\|f\|_{L_{1}} .
$$

\section{Appendix A. Proof of lemma 1.}

Let us consider some $x$ inside $Q_{0}=[0,1]^{d}$. Then the proposition holds true not only for infinite measure $\mu^{d}$ but for $\left.\mu^{d}\right|_{Q\left(x, r_{0}\right)}$ where $r_{0}>s_{0}-u$ or more explicitly $r_{0}=\rho d^{1 / 4}$, $r_{0} \in \mathbb{Z}$ as well, with some appropriate $\rho$ independent of $d$. Thus for a significantly large cube $K(R)$ the proposition will hold true for any translated unit cube $Q_{0}+n$ where $n \in \mathbb{Z}^{d}$ within a smaller cube $K\left(R-r_{0}\right)$ with the same center as $K(R)$. The number of unit cubes that fits into $K\left(R-r_{0}\right)$ is $N=\left(R-r_{0}\right)^{d}$.

Thus asymptotically as $d \rightarrow \infty$

$$
\frac{N}{(R+1)^{d}} \sim\left(1-\frac{\left(r_{0}-1\right)}{(R+1)}\right)^{d} .
$$

If we now set $R \geq \theta d^{5 / 4+\varepsilon}$ where $\varepsilon>0$ and $\theta$ is some constant independent of $d$, then asymptotically when $d \rightarrow \infty$ we will obtain

$$
\left(1-\frac{\left(r_{0}-1\right)}{(R+1)}\right)^{d} \geq\left(1-\frac{\rho}{\theta d^{1+\varepsilon}}\right)^{d} \sim 1 .
$$

Now taking into account the fact that $N<(R+1)^{d}$ we arrive at the desired result

$$
\lim _{d \rightarrow \infty} \frac{N}{(R+1)^{d}}=1 \text {. }
$$




\section{Appendix B. Proof of Lemma 2.}

Although the original proof of this Lemma can be found in [3] we give it here for completeness and unification of notations.

Suppose $\mu=\sum_{i=1}^{n} \delta_{x_{i}}$ and $\alpha>0$ are such that the inequality

$$
\alpha|\{M \mu \geq \alpha\}|>c n
$$

holds. Let us prove that for every $\epsilon>0$ there exists $f \in L^{1}\left(\mathbb{R}^{d}\right)$ with $\|f\|_{1}=n$ and $\{M \mu \geq \alpha\} \subset\left\{M f>(1+\epsilon)^{-1} \alpha\right\}$. Let $l(Q)$ denotes the side length of the cube $Q$. For each $x \in\{M \mu \geq \alpha\}$, select a cube $Q(x, r)$ with

$$
\frac{N(Q(x, r))}{|Q(x, r)|}>\alpha \text {. }
$$

Here $N(Q(x, r))$ stands for a number of point masses contained in $Q(x, r)$.

We make additional assumption that $l(Q(x, r)) \geq \alpha^{-1 / d}$. This is always possible to fulfill since each $Q(x, r)$ must contain at least one point mass. Since $\alpha$ is strictly positive we can define $a$ as

$$
a=\sup _{r}\{l(Q(x, r)): x \in\{M \mu \geq \alpha\}\}<\infty .
$$

Given any $\epsilon>0$, choose $\delta(\epsilon)$ such that for every $y \in\left[\alpha^{-1 / d}, a\right],(y+\delta)^{d} / y^{d}<1+\epsilon$. Let $E_{i}$ be a closed cube centered at $x_{i}$ with the sidelength $\delta$ and define $f=\delta^{-d} \sum_{1}^{n} \chi_{E_{i}}$. Replacing each $Q(x, r)$ by the cube $Q(x, r+\delta / 2)$, we see that $\{M \mu \geq \alpha\} \subset\{M f>$ $\left.(1+\epsilon)^{-1} \alpha\right\}$.

Pick $\epsilon>0$ so small that $\alpha|\{M \mu \geq \alpha\}|>(1+\epsilon)$ cn. Since $\|f\|_{1}=n$, we get

$$
\alpha\left|\left\{M f \geq \alpha(1+\epsilon)^{-1}\right\}\right| \geq \alpha|\{M \mu \geq \alpha\}|>(1+\epsilon) c|| f \|_{1} .
$$

The conclusion now follows by setting $\beta=\alpha(1+\epsilon)^{-1}$.

\section{References}

[1] E.M. Stein and J.-O. Strömberg, Behavior of maximal functions in $\mathbb{R}^{n}$ for large $n$, Ark. Mat. 21(2) (1983), 259-269. MR727348.

[2] J.M. Aldaz, The weak type $(1,1)$ bounds for the maximal function associated with cubes grow to infinity with the dimension, Ann. Math. 173 (2011), 1013-1023.

[3] J.M. Aldaz, A remark on the centered n-dimensional Hardy-Littlewood maximal function, Czechoslovak Math. J. 50(125)(1) (2000), 103-112.

[4] M. Abramowitz and I. Stegun, Handbook of Mathematical Functions, Dover Publ. Inc., New York, 1972.

Institutionen för matematik, Kungliga Tekniska Högskolan, Department of MathematICS, KTH, SE-100 44, Stockholm, Sweden

E-mail address: iakovlev@kth.se

Institutionen för matematik, Kungliga Tekniska Högskolan, Department of MathematICS, KTH, SE-100 44, Stockholm, Sweden

E-mail address: jostromb@kth.se 